\title{
Acute Respiratory Distress Syndrome Following Anaphylactic Shock-“A Deadly Duel"-Case Report and Literature Review
}

\author{
Ungamandadige P. M. Fernando ${ }^{1}$, Malmee P. Dharmawardhane1, Nishanthan Subramaniam², \\ Srisothinathan Nimalan², Kukulagoda U. I. S. Gunathilake3, Baththirange M. Munasinghe ${ }^{{ }^{*}}$ \\ ${ }^{1}$ National Blood Transfusion Service, District General Hospital, Mannar, Sri Lanka \\ ${ }^{2}$ Anaesthesiology and Intensive Care, District General Hospital, Mannar, Sri Lanka \\ ${ }^{3}$ Clinical Medicine, Post Graduate Institute of Medicine, University of Colombo, Colombo, Sri Lanka \\ ${ }^{4}$ District General Hospital, Mannar, Sri Lanka \\ Email: pasindu9091@gmail.com,nishanthan@royal.net, sri.nimalan1988@gmail.com, \\ inokashyamali4@gmail.com, ${ }^{\star}$ malakafmp@gmail.com
}

How to cite this paper: Fernando, U.P.M., Dharmawardhane, M.P., Subramaniam, N., Nimalan, S., Gunathilake, K.U.I.S. and $\mathrm{Mu}-$ nasinghe, B.M. (2021) Acute Respiratory Distress Syndrome Following Anaphylactic Shock-“A Deadly Duel”-Case Report and Literature Review. Open Journal of Anesthesiology, 11, 33-38.

https://doi.org/10.4236/ojanes.2021.112004

Received: January 3, 2021

Accepted: February 2, 2021

Published: February 5, 2021

Copyright $\odot 2021$ by author(s) and Scientific Research Publishing Inc. This work is licensed under the Creative Commons Attribution International License (CC BY 4.0).

http://creativecommons.org/licenses/by/4.0/ (c) (i) Open Access

\begin{abstract}
A 58-year-old Asian female developed acute respiratory distress syndrome (ARDS) following anaphylactic shock. Several similar cases have been reported in the literature, attributed to the pathophysiological mechanisms of anaphylaxis or interestingly, the treatment itself; adrenaline, majority of the latter being related to administration of supra-therapeutic doses. According to our clinical experience, the possibility of ARDS should be considered in patients who develop unexplainable hypoxaemia following anaphylactic shock. This case report discusses the pathophysiology of ARDS both in anaphylaxis and following epinephrine treatment and key aspects of management of ARDS with a main focus on the role of high flow oxygen, diuretics and anxiolytics. The importance of avoiding drug administration errors is also highlighted.
\end{abstract}

\section{Keywords}

Acute Respiratory Distress Syndrome (ARDS), Anaphylaxis,

Epinephrine/Adrenaline, Pulmonary Edema

\section{Introduction}

Anaphylaxis, a relatively common life threatening medical emergency, is known to cause acute respiratory distress syndrome (ARDS). Similarly, the treatment of choice in anaphylactic shock, epinephrine, is also implicated in non-cardiogenic pulmonary oedema and ARDS, although the latter is rare and associated with 
supratherapeutic doses in general. The possible mechanisms of the pulmonary oedema in each of these instances are discussed in detail in this paper. Special emphasis is given to the management of both anaphylaxis-based and adrenaline induced pulmonary oedema. It is important to note that high index of suspicion and early diagnosis are paramount in avoiding any acute or long-lasting effects following such an episode. The necessity of preventing iatrogenic drug administration errors is also discussed.

\section{Case Presentation}

A 58-year-old diabetic woman for twelve years was managed in the surgical ward of our institution for lower limb cellulitis. Sensitivity test for crystalline penicillin was positive. She was started on Meropenum $500 \mathrm{mg}$ IV as per local protocol. Her blood and tissue cultures were negative. Cellulitis was settling by day 05 . The next day, the patient was accidentally injected with parenteral penicillin due to a nursing error. Soon after, her BP crashed to 76/30 with a tachycardia of 140 and a peripheral saturation of $86 \%$. Examination revealed clear lung bases with no stridor. Immediate working diagnosis of anaphylaxis was made and IM epinephrine (1:1000) $0.5 \mathrm{mg}$ to the anterolateral thigh and intravenous crystalloid $10 \mathrm{ml} / \mathrm{kg}$ bolus was administered while monitoring vitals. She was haemodynamically stable following 6 cycles of epinephrine and transferred to the ICU. Inward chest X-ray, ECG and echocardiogram revealed no abnormalities. ABG obtained in the 1st hour was normal ( $\mathrm{pH} 7.37, \mathrm{PaO}_{2} 96 \mathrm{mmHg}$, $\mathrm{PaCO}_{2} 34 \mathrm{mmHg}$, lactate $1.6 \mathrm{mmol} / \mathrm{dl}, \mathrm{FiO}_{2}$ 0.4). Blood for serum tryptase was collected although testing was not done due to the unavailability of the reagents.

Four hours later, the patient developed worsening hypoxaemia with bi-basal fine crepitation. Her NIBP was $144 / 84 \mathrm{mmHg}$ with a pulse rate of $110 / \mathrm{min}$. ABG revealed a $\mathrm{PaO}_{2}$ of $60 \mathrm{mmHg}$ with $15 \mathrm{l} / \mathrm{min}$ of $\mathrm{O}_{2}$ via face mask $(\mathrm{P} / \mathrm{F}$ ratio $=67)$. $\mathrm{CPAP}$ ventilation was commenced. Intravenous Frusemide and morphine were administered simultaneously. CXR showed bilateral non-homogenous infiltrates indicating acute respiratory distress syndrome (ARDS) (Figure 1).

Ultrasound of the chest revealed increased number of heterogeneous " $\mathrm{B}$ " lines. ECG showed no acute ischaemic changes. 2D echocardiogram revealed an EF of $60 \%$, normal LV/RV filling and contractility. Inferior vena caval (IVC) diameter and the caval index were normal refuting the possibility of fluid overload. Her serum blood sugar levels were normal throughout. ABG repeated 2 hours after initial stabilization showed a $\mathrm{pH}$ of 7.38, $\mathrm{PCO}_{2} 42 \mathrm{mmHg}, \mathrm{PO}_{2} 150 \mathrm{mmHg}$ and $\mathrm{HCO}_{3}^{-} 24 \mathrm{mmol} / \mathrm{l}$ and lactate of $1.5 \mathrm{mmol} / \mathrm{l}(\mathrm{P} / \mathrm{F}=150)$. Maintenance fluids (1 $\mathrm{ml} / \mathrm{kg} / \mathrm{hr}$ ) were infused while monitoring urine output. She made a steady recovery over the next 48 hours, weaned from the ventilator and put on $\mathrm{O}_{2}$ via face mask. On Day 4 of ICU stay she was transferred to the ward. She was discharged home 3 days later and followed up in surgical clinic for wound management. Considering the nature of onset and causality, diagnosis of ARDS subsequent to anaphylaxis was made. 


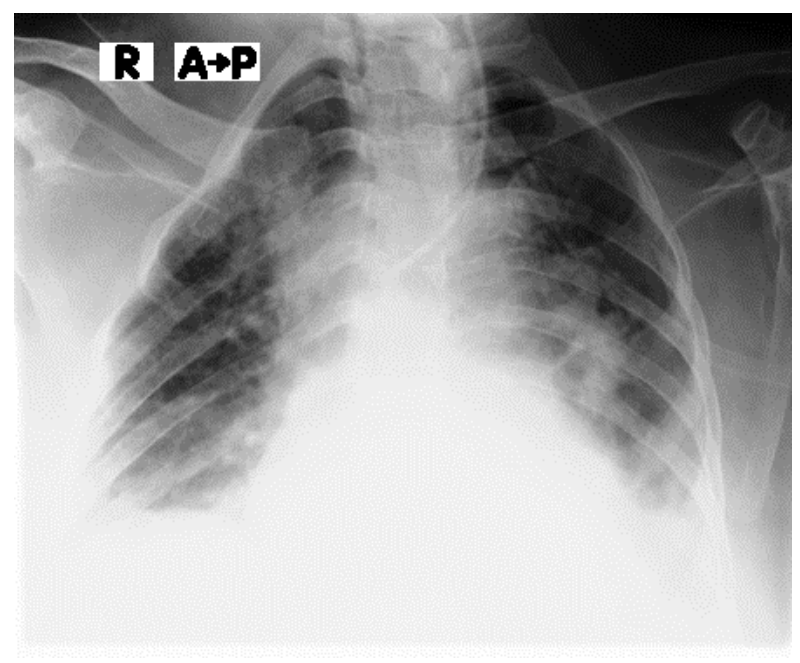

Figure 1. AP CXR taken 6 hours after the onset of anaphylaxis showing bilateral patchy infiltrates indicating setting ARDS.

\section{Discussion}

The multisystem involvement with the propensity for organ dysfunction could lead to serious morbidity and mortality in anaphylaxis. A significant number of patients who develop lower airway respiratory symptoms may ultimately develop acute respiratory distress syndrome (ARDS).

There are varying clinical disorders which may lead to ARDS, although the pathogenesis in anaphylaxis is considered to be a widespread inflammatory injury to lung endothelium and epithelium causing marked rise in permeability, permitting exudation into alveoli. [1] The resulting injury further promotes the inflammatory cascade leading to extensive injury to lung parenchyma precipitating respiratory insufficiency. Anaphylaxis due to morphine which is secondarily complicated by ARDS is previously reported by Tobi, et al. (2018) [2] in a 16 year old patient who underwent an orchidopexy under spinal anaesthesia supplemented by intravenous morphine. He developed a biphasic reaction attributed later to morphine, which was complicated by severe ARDS. The patient was intubated and ventilated for few days but recovered completely. The ARDS in this particular case had occurred immediately following anaphylactic shock. In our patient, intravenous penicillin injection resulted in anaphylactic shock (managed with a relatively low cumulative dose of intramuscular epinephrine) and subsequent ARDS. Several animal studies have shown that the onset of pulmonary edema can be as early as one hour or less [3] although signs of ARDS were apparent after about 4 hours in our patient. She had stable haemodynamics thus was managed with non-invasive ventilation and Oxygen therapy in the Intensive care and made a slow but steady recovery. With regard to the mechanisms of ARDS in anaphylaxis, it could be either by direct injury to the lung parenchyma or secondary to effects of systemic mediators on the lung, released during anaphylaxis. Out of the indirect causes of ARDS in anaphylaxis, the commonest is sepsis leading up to $40 \%$ of cases [4]. Varying degrees of ARDS 
precipitated by allergic reactions, have been reported in several other singular case reports. These were associated with different classes of drugs ranging from contrast-media to cases where aetiology not being concluded [5] [6].

Numerous case reports on pulmonary edema following administration of epinephrine have been reported in the literature albeit the majority is linked to supra-therapeutic doses [7] [8] [9]. Several mechanisms are postulated, among which the commonly accepted are: 1) increased peripheral vascular resistance and reduced diastolic filling time. This leads to increased LV pressure and in turn elevated back pressure on pulmonary vessels; 2) disturbed Starling forces at the level of pulmonary capillaries due to pulmonary vasoconstriction and/or increased left atrial pressure leading to increased pulmonary arterial pressure; 3 ) shifting of blood from systemic to pulmonary circulation due to increased peripheral vasoconstriction; and 4) additional central neurogenic role that leads to pulmonary edema following massive doses of epinephrine. [10] Wang, N.S., et al. (1971) reported ultra-structural nonspecific toxic injury changes in pulmonary type I and II epithelial cells and Clara cells following epinephrine injection in mice which may be contributory to the pulmonary edema. [11] Beate Rassler (2012) has demonstrated that pulmonary edema ensues more rapidly with $\alpha$ rather than $\beta$ adrenergic stimulation. [3] This suggests that a patient who is on $\beta$ blocking agents may be at elevated risk of developing pulmonary edema due to unopposed $\alpha$ action of epinephrine. Systemic hypotension following the initial hypertension is characteristically reported in literature as the "diphasic response of epinephrine" [12]. This is assumed to be due to prolonged stimulation of $\beta$ adrenoceptors after the cessation of $\alpha$ adrenoceptor stimulation subsequent to the catabolism of exogenous epinephrine below a certain plasma threshold level which may be propagated by the release of epinephrine first taken up by the nerve endings. This could be treated with intravenous crystalloids and if warranted, with inotropes, along with strict monitoring of fluid balance.

High flow oxygen combined with positive pressure ventilation is the first line therapy in ARDS. [9] The elevated pressures in broncho-alveolar system counteract the pressure in pulmonary capillaries and minimize transudation. [13] Non-invasive ventilation (NIV) could be beneficial to patients with haemodynamic stability and mild to moderate degrees or even severe ARDS where a favourable response to NIV is witnessed. Mechanical ventilation following endotracheal intubation is opted in cases of associated multi-organ dysfunction or severe, refractory cases of ARDS. Historically the use of morphine has been established in the treatment of pulmonary edema. Alleviation of anxiety dampening the sympathetic over activity, overall reduction in metabolic rate and reduced venous pressure rendering less workload on the heart, reduced suction effect on the lungs due to central depressor effects are the possible pharmacodynamics of morphine. [9] Aim of diuretics in non-cardiogenic pulmonary edema is to promote a rapid diuresis and remove excess fluids. Rarely this could be adequate as standalone therapy [9]. Ersoz and Finestone (1971) proposed the 
place of intravenous chlorpromazine in the management of ARDS following anaphylaxis [10] although this is no longer practiced.

Error in drug administration is a well-known cause for increased in-patient morbidity and mortality. Adhering to the "five rights" and developing local protocols are indispensable in minimizing these errors. Using more than one identifier such as name with initials, age or guardian's name would be decisive in avoiding possible misidentification of patients as was the case in our patient. Last but not least, we suggest that additional safety measures such as, displaying a sign board, educating the patient or caregiver to notify any allergies and proper handing over, particularly of allergy history should be followed specifically in developing countries where digital patient management systems are unavailable.

\section{Conclusion}

Anaphylaxis is notorious for its animosity in both the rapidity of onset and poorer outcomes in cases of delayed treatment. The definitive treatment for anaphylaxis and epinephrine could also lead to life threatening complications such as flash pulmonary edema and ARDS. Thus, monitoring a post anaphylactic shock patient in a high dependency unit is highly advisable. Anaphylaxis in hospitalized patients is almost always due to erroneous drug administration. Proper patient identification using several identifiers, following the "five rights" and adhering to local protocols are pivotal in avoiding these errors.

\section{Acknowledgements}

Dr. Thamara Hapuarachchi, Consultant Clinical Microbiologist, District General Hospital, Mannar, for her kind contribution.

\section{Funding}

No funding was available for the preparation of the manuscript.

\section{Author Contributions}

M.M., N.S. and S.N. managed the patient. P.F. wrote the manuscript with the contribution from M.D., I.G., M.M., N.S. and S.N. M.M. supervised the compilation of the manuscript and the publication process. All reviewed and approved the final manuscript.

\section{Consent}

Informed, written consent was taken from the patient for the inclusion of clinical details and the investigation reports.

\section{Ethical Clearence}

Not required. 


\section{Conflicts of Interest}

The authors declare no conflicts of interest.

\section{References}

[1] Matthay, M.A. and Zemans, R.L. (2011) The Acute Respiratory Distress Syndrome: Pathogenesis and Treatment. Annual Review of Pathology, 6, 147-163. https://doi.org/10.1146/annurev-pathol-011110-130158

[2] Tobi, K.U., et al. (2018) Acute Respiratory Distress Syndrome Following a Biphasic Anaphylactic Reaction to Morphine: A Case Report and Review of the Literature. Southern African Journal of Anaesthesia and Analgesia, 24, 165-167. https://doi.org/10.1080/22201181.2018.1529856

[3] Rassler, B. (2012) Contribution of Adrenergic Mechanisms to the Development of Pulmonary Edema. Scientifica, 2012, Article ID: 829504.

https://doi.org/10.6064/2012/829504

[4] Fein, A.M., Calalang-Colucci, M.G. (2000) Acute Lung Injury and Acute Respiratory Distress Syndrome in Sepsis and Septic Shock. Critical Care Clinics, 16, 289-317. https://doi.org/10.1016/S0749-0704(05)70111-1

[5] Park, J., Byun, I.H., Park, K.H., et al. (2015) Acute Respiratory Distress Syndrome after the Use of Gadolinium Contrast Media. Yonsei Medical Journal, 56, 1155-1157. https://doi.org/10.3349/ymj.2015.56.4.1155

[6] Efeturi, A.T., Lily, M.I., Adebowale, A., et al. (2017) Anaphylaxis Complicated by Acute Respiratory Distress and Fatal Outcome in a Nigerian Family. Journal of Clinical and Diagnostic Research, 11, SD01-SD03.

[7] Novey, H.S. and Meleyco, L.N. (1969) Alarming Reaction after Intravenous Administration of $30 \mathrm{ML}$ of Epinephrine. JAMA, 207, 2435-2436.

https://doi.org/10.1001/jama.1969.03150260095018

[8] Vestergaard, L. (1993) Pulmonary Edema after Intravenous Injection of Eyedrops Containing Adrenaline. Ugeskr Laeger, 155, 3776-3777.

[9] Luisada, A.A. and Cardi, L. (1956) Acute Pulmonary Edema; Pathology, Physiology and Clinical Management. Circulation, 13, 113-135.

https://doi.org/10.1161/01.CIR.13.1.113

[10] Ersoz, N. and Finestone, S.C. (1971) Adrenaline-Induced Pulmonary Oedema and Its Treatment. A Report of Two Cases. British Journal of Anaesthesia, 43, 709-712. https://doi.org/10.1093/bja/43.7.709

[11] Wang, N.S., et al. (1971) Ultrastructural Changes of Clara and Type II Alveolar Cells in Adrenalin-Induced Pulmonary Edema in Mice. The American journal of pathology, 62, 237-252.

[12] Carter, B.T., et al. (1971) Severe Reaction to Accidental Subcutaneous Administration of Large Doses of Epinephrine. Anesthesia \& Analgesia, 50, 175-178. https://doi.org/10.1213/00000539-197103000-00002

[13] Barach, A.L., Martin, J. and Eckman, M. (1938) Positive Pressure Respiration and Its Application to the Treatment of Acute Pulmonary Edema. Annals of Internal Medicine, 12, 754-795. https://doi.org/10.7326/0003-4819-12-6-754 